\title{
PERSISTENCIA DE ERRORES CONCEPTUALES EN EL ESTUDIO DEL EQUILIBRIO QUIMICO
}

\author{
CARLOS J. FURIO MAS \\ ELENA ORTIZ \\ (I.C.E. de la Universidad de Valencia)
}

\section{SUMMARY}

This paper exposes the existence of certain conceptual errors in the study of chemical equitibrium, lack of differentiation between full chemical reaction and equilibrium compartimentation of equilibrium,... and, in particular, the strong persistance of the error of mass-concentration of substances. For it, examples were taken from two different groups: Pre-University course students and teachers students.

\section{INTRODUCCION}

El interès del estudio de los errores conceptuales que se nianifiesta en la aparición reciente de numerosos trabajos en revistas de investigacion (Nussbaum, 1981) y jornadas didácricas (Carrascosa y Gil, 1981) (Carrascosa y Gil, 1982), radica en la necesidad de su conocimiento por eł profesorado para abordar con cierta efi. cacia el proceso de ensenanza-aprendizaje.

La actualidad del estudio de los errores conceptuales en el campo del equilibrio quimico viene confirmada por la tercera recomendación del grupo de discusion que, sobre este tema, coordino el profesor D. Vitorovic (1979) en la Third International Conference on Chemical Education celebrada en Dublin y que sugiere literaimente:

"Deberian organizarse trabajos experimentales sobre equivocaciones y errores conceptuales de los estudiantes, a consecuencia de los diferentes métodos de ensenanza (del equilibrio químico)".

En el presente trabajo tratamos de verificar la persisrencia de algunos errores conceptuales dentro del estudio del equilibrio químico, incluso en alumnos que hacen el curso para obtener el certificado de aptittrd pedagogica (C A P), diploma necesario para opositar a una plaza de profesor según la tegislación vigente, y que, en su mayoria, es cursado por licenciados universitarios. La gran persistencia de estos errores conceptuales significa, a nuestro entender, el desconocimiento por el profesorado de los esquemas conceptuales de los alumnos, no logrando las diversas metodologias de enseñanza empleadas el cambio conceptual en el alumnado. Cambio conceptual que ha sido propugna. do desde hace bastante tiempo por relevantes epistemologos (Bachelard, 1938).

\section{PLANTEAMIENTO Y OBJETIVO DEL TRABAJO}

Es frecuente observar la aparición de reiterados errores conceptuales en los alumnos que los profesores solenos creer que son pequetos fallos debidos a la incom. prension, por parte del estudiante, de los conceptos que se explican o se aplican en la clase ordinaria. Ests justificaciones del profesorado ocultan, en reaiidad, su ignorancia de las causas de la persistencia y existencia de estos errores conceptuales. Si admitimos como hipótesis de trabajo que estos errores son como los icebergs que afforan de una solida y coherente estructura cognoscitiva del alumnado, comprenderemos que el origen de aquellos habrá que buscartos en el cuerpo de conocimientos - preconceptos, conceptos o ideasque el alumnado ha asimilado, intercambiado o captado a lo largo de sus viviencias académicas $y / 0$ extracadémicas. (Vygosky, 1973) (Ausubel, 1978) (Carrascosa, Gil y González, 1982).

La diagnosis de lo que piensan los alumnos en el campo de la Física y de la Quimica, está siendo abordada en recientes trabajos de investigación educativa y el nuestro tiene como objetivo fundamental comprobar que existen determinados errores conceptuales cuya persistencia, incluso a niveles estudiantiles elevados, es un sintoma de que la estructura cognoscitiva del alumnado no ha sido afectada por la ensentanza recibida. De forma que el alumno conserva intacto su esquema conceptual inicial ya que las metodologías empleadas no han servido para plantear el debate interior entre la vieja visión del comportamiento de la materia y el nuevo paradigma científico que se pretendfa intercambiar en la clase.

$\mathrm{Si}$, por otra parte, aceptamos como tesis la existencia 
de un cierto paralelismo entre el desarrollo intelectual del atumno y el crecimiento de la producción cientifica, como ha sido mostrado por Piaget (1975), existe una base de partida para analizar las estructuras cognoscitivas del alumnado, viendo como se ha verificado el progreso cientifico (Kuhn, 197!) y revisando cual era el pensamiento de la comunidad cientifica en épocas pasadas, ya que sus conclusiones serian las consecuencias logicas de sus ideas y, en definitiva, de su visión científica del problema.

En este trabajo estudiaremos, entre otros, el llamado error conceptuat "masa-concentración", tratando de comprobar si persistia no sólo en alumnos de COU que habian estudiado recientemente el tema del equilibrio quimico, sino también en licenciados que hacia bastante tiempo que habian tratado el mismo. Ello nos demostraria que la causa de este error habrá que buscarla no en aspectos anecdóticos, sino en una raíz más profunda; en la forma de interpretar el comportamiento quimico de la mateta por los estudiantes de Quimica.

En resumen, el objetivo del trabajo consiste en corroborar la existencia de determinados errores conceptuales sobre ef equitibrio quimico que han sido tratados por otros autores (Johnstone, Mac Donald y Webb, 1977) (Wheeler y Kass, 1978) (Teterin, 1979) y fundamentalmente verificar la fuerte persistencia del error "masa-concentración» en alumnos que por sus conocimientos, al menos en teoria, no to debian cometer.

\section{PROCEDIMIENTO}

Con el fin de verificar la persistencia de algunos errores conceptuales en el equitibrio químico, se encuestó por una parte a dos grupos de alumnos del Curso de Orientacion Universitaria (COU) y por otra, a oiros dos grupos de alumnos del CAP, que pueden ser consideradas, a priori, muestras de diferentes poblaciones. A continuación se detalla el material suministrado y los alumnos encuestados.

\subsection{Material suministrado}

Como interesaba contrasiar la existencia o no de determinados errores conceptuales detectados en la bibliografía como ya se ha indicado anteriomente, se confecciono un conjunto de items, uno para cada uno de los errores, todos ellos cerrados, de opcion múltiple, incluida la respuesta "no lo sé" para evitar la aleatoriedad en las contestaciones. El alumno, después de elegir la respuesta, debía razonar brevemente su contestacion, con el fin de detectar, en lo posible, cuales eran las ideas en que basaba aqueila. Los enunciados de estos items se encuentran en el Apéndice. Para el enun. ciado de los items se pensó en distractores adecuados para los objetivos buscados.

Los errores fueron los siguientes:

Item $\pi^{\circ} 1$ : Desconocimiento de las condiciones que debe reunir un sistema quimico, en el que intervienen gases, para poder iniciar el estudio del equilibrio quinico (sistema cerrado).

Item $n^{a}$ 2: Concepción del estado de equilibrio como dos estados, atribuidos a los reaccionantes y a los productos respectivamente (Compartimentación del equilibrio).

Item $n^{\circ}$ 3: Se concibe la constante de equilibrio como una función o como una constante a la misma temperatura? (No constancia de la constante).

Item $n^{\circ}$ 4: El error "masa-concentracion" -si bien, la masa venía medida en moles de sustancia-. Es decir, el alumno considera de forma automática que el número de moles de sustancia es la concentración.

Item $n^{\circ}$ 5: Equivalencia mental entre la frase ula concentración de sólido" y la frase "la masa de sólidon, que vendría a constatar nuevamente el error masa-concentración (item 4) aunque por otra via.

\subsection{Alumnos encuestados}

Los dos grupos de alumnos de COU (35 y 26 alumnos) fueron encuestados por uno de los autores del trabajo, cuando habia acabado el estudio del tema «Equilibrio quimico», dándoles tiempo suficiente, sin limitaciones, para poder concluir con tranquilidad sus contestaciones.

En cuanto a los dos grupos de alumnos del CAP que fueron encuestados, hay que indicar que el tema de los errores conceptuales formaba parte de su programa de Didáctica de Química y la encuesta se hizo el primer dia de clase, sin previo aviso. Después de encuestados se hizo la oportuna discusión sobre el tema, llegando a la conclusión de la necesidad de incluirlo en los programas de ensenanza de los futuros profesores. Respecto al espectro de especialidades y niveles de estos alumnos en el momento de la encuesta, se indican a continuacion:

Alumnos del C A P encuestados

\begin{tabular}{ccccccc} 
Grupo & $\begin{array}{c}\text { Ldios. } \\
\text { Quim. }\end{array}$ & Fis. & $\begin{array}{c}\text { Estud. } \\
5^{\circ} \text { Quim. }\end{array}$ & $\begin{array}{c}\text { Estud. } \\
5^{\circ}\end{array}$ Fis. & $\begin{array}{c}\text { 1dos. } \\
\text { Frm. }\end{array}$ & TOTAL \\
\hline 1 & 13 & 6 & 8 & 1 & 5 & 28 \\
2 & 13 & - & 10 & 1 & 3 & 27 \\
\hline TOTAL & 26 & 6 & 18 & 2 & 3 & 55
\end{tabular}


Todos estos alumnos habian estudiado en su carrera el tema del equiliorio quimico, si bien en los primeros cursos. Es decir, hacia como minimo tres años que lo habian tratado.

\section{RESULTADOS: ANALISIS Y DISCUSION}

En la tabla 1 se presentan los resultados baltados al encuestar por una parte a los alumnos de COU (grupo A) y por otra a los alumnos del CAP (grupo B). Junto a los porcentajes totales de alumnos equivocados (TOT.EQ). se incluyen las desviaciones estandard $s$ de cada uno de aquellos que, segun Garret (197!) vienen dadas por la expresion:

$$
s=\frac{\sqrt{p(100-p)}}{N}
$$

donde $\rho$ representa el porcentaje total de desaciertos y $N$, el numero de alumnos encuestados. Dado que el trabajo se considera preliminar, no se ha calculado el factor z para valorar las diferencias significativas de forma rigurosa. No obstante, se comentan de manera cualitativa los resultados encontrados. En algunos casos se ha procedido al análisis de las respuestas incorrectas mayoritarias que proporcionará una idea de los errores mas frecuentes del alumnado, dnetro de las opciones planeadas por los attores. Tengase en cuenta que al ser un cuestionario cerrado, las respuestas resuttan necesariamente condicionadas.

Un rápido análisis global de la tabla I hace ver la elevada cantidad de alumnos de COU en los que persisten errores fundamentales, a pesar de haberse impartido recientemente el tema del equitibrio quimico (se les encuestó nada más concluirlo). Es más, incluso en algunos prerrequisitos como es la consideración de cerrar el sistema químico para poder abordar el estudio de un equilibrio, hay casi la mitad de alumnos de la muestra (45.7) que no ha sabido distinguir aquella situación en aquel sistema evolucionaria hacia un equilibrio (item $n^{\circ} 1$ ). Similr consecuencia puede extraerse de las respuestas dadas por los alumnos de COU al ttem $\mathrm{n}^{\circ} 2$, cosa que ya preveiamos, de los equivocados (un $53 \%$ ) la mayoria ( $35.2 \%$ ) considera que en el equilibrio hay dos estados atribuidos, uno a los reaccionantes - primer miembrode la ecuación química- y otro, a los productos -segundo miembro de aquella ecuacion-. Hohnstone, McDonald y Webb (1977) obiuvieron un porcentaje más alco $(79 \%)$ en alumnos de similar nivel educativo. Respecto a la no constancia de la constante de equilibrio sin alterar la temperatura, parece excesiva la cantidad de alumnos de COU $(95 \%)$ que se equivocan y, a través de sus contestaciones, se puede constatar la utilización de la expresión de la constante de equilibrio en forma memoristica, ya que para ellos la $\mathrm{K}$ no es una constante, sino una función matemática que depende de determinadas variables

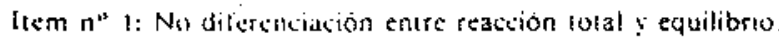

\begin{tabular}{|c|c|c|c|c|c|c|c|c|}
\hline \multirow{2}{*}{ Girupo } & \multirow{2}{*}{$N$} & \multicolumn{5}{|c|}{$\%$ RESPLIESTAS } & \multirow[b]{2}{*}{ TOT. EQ. } & \multirow[b]{2}{*}{$s$} \\
\hline & & la & $1 \mathrm{~b}$ & 16 & 10 & le & & \\
\hline$\hat{A}$ & $\begin{array}{l}61 \\
55\end{array}$ & $\begin{array}{l}9.8 \\
5.5\end{array}$ & $\begin{array}{r}23.0 \\
2.0\end{array}$ & $\begin{array}{l}52.5 \\
81.8\end{array}$ & $\begin{array}{l}9.8 \\
3.6\end{array}$ & $\begin{array}{l}4.9 \\
7.2\end{array}$ & $\begin{array}{l}47.5 \\
18.2\end{array}$ & $\begin{array}{l}6.4 \\
5.2\end{array}$ \\
\hline
\end{tabular}

ftem n* 2: Compartimentación del equiliorio.

\begin{tabular}{|c|c|c|c|c|c|c|c|c|}
\hline \multirow{2}{*}{ Girupo } & \multirow{2}{*}{$N$} & \multicolumn{5}{|c|}{$\%$ RESPLIFSTAS } & \multirow[b]{2}{*}{ TOT. EQ. } & \multirow[b]{2}{*}{$s$} \\
\hline & & $2 a$ & 26 & $\pi$ & $2 d^{\circ}$ & $2 c$ & & \\
\hline $\begin{array}{l}A \\
13\end{array}$ & $\begin{array}{l}54 \\
55\end{array}$ & $\begin{array}{l}3.7 \\
3.6\end{array}$ & $\begin{array}{l}7.4 \\
0\end{array}$ & $\begin{array}{l}35.2 \\
18.2\end{array}$ & $\begin{array}{l}46.3 \\
74.5\end{array}$ & $\begin{array}{l}7.4 \\
3.6\end{array}$ & $\begin{array}{l}53.7 \\
25.5\end{array}$ & $\begin{array}{l}6.8 \\
5.9\end{array}$ \\
\hline
\end{tabular}

Item 3: No constancia de la constante de equilibrio.

\begin{tabular}{|c|c|c|c|c|c|c|c|c|}
\hline \multirow{2}{*}{ Grupo } & \multirow{2}{*}{$N$} & \multicolumn{5}{|c|}{ \% RESPUESTAS } & \multirow[b]{2}{*}{ TOT. EQ. } & \multirow[b]{2}{*}{$s$} \\
\hline & & $3 \mathrm{a}$ & 36 & $3 c$ & $30^{*}$ & $3 e$ & & \\
\hline $\begin{array}{l}A \\
B\end{array}$ & $\begin{array}{l}61 \\
55\end{array}$ & $\begin{array}{r}11.5 \\
7.3\end{array}$ & $\begin{array}{l}77.0 \\
14.5\end{array}$ & $\begin{array}{l}4.9 \\
3.6\end{array}$ & $\begin{array}{r}4.9 \\
72.7\end{array}$ & $\begin{array}{l}1.6 \\
1.8\end{array}$ & $\begin{array}{l}95.1 \\
27.3\end{array}$ & $\begin{array}{l}2.8 \\
6.0\end{array}$ \\
\hline
\end{tabular}

item $n^{\circ}$ 4: Error masa = concentracion.

\begin{tabular}{|c|c|c|c|c|c|c|c|}
\hline \multirow{2}{*}{ Grupo } & \multirow{2}{*}{$N$} & \multicolumn{4}{|c|}{ \%O RESPLIESTAS } & \multirow[b]{2}{*}{ TOT. EQ. } & \multirow[b]{2}{*}{$\mathrm{s}$} \\
\hline & & $43^{\circ}$ & $4 b$ & $4 c$ & 40 & & \\
\hline $\begin{array}{l}A \\
8\end{array}$ & $\begin{array}{l}61 \\
55\end{array}$ & $\begin{array}{l}21.3 \\
40.0\end{array}$ & $\begin{array}{l}0 \\
5.5\end{array}$ & $\begin{array}{l}77.0 \\
54.5\end{array}$ & $\begin{array}{l}1.6 \\
0\end{array}$ & $\begin{array}{l}78.7 \\
60.0\end{array}$ & $\begin{array}{l}5.2 \\
6.6\end{array}$ \\
\hline
\end{tabular}


liem n० 5: Error inasa = concentracion (en solidos)

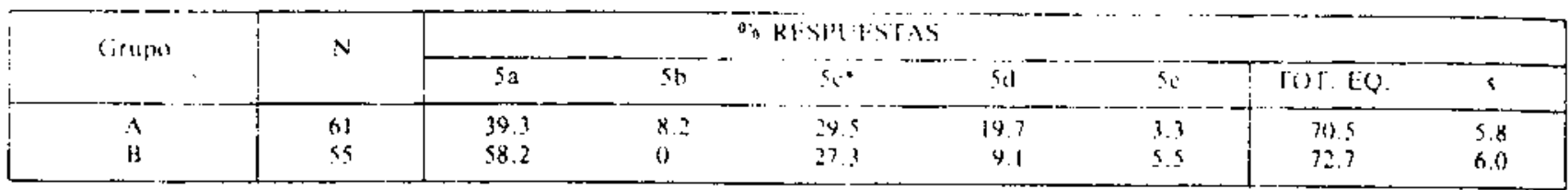

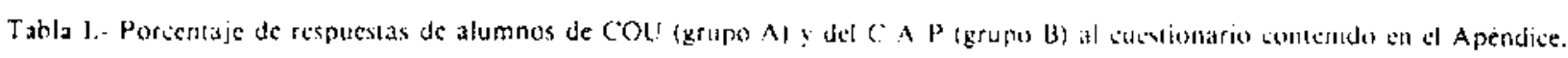

Como era de esperar, en estos tres errores conceptuales hay diferencias significativas entre los resultados encontrados en alurnnos de COU y de CAP, debido a la mayor experiencia curricular de estos últimos. Sin embargo, al comparar los resultados hallados en el error masa-concentración (item $n^{\circ} \S$ ), puede apreciarse que las diferencias entre estas dos muestras ya no son tan grandes (78.7 y $60 \%$ respectivamente) y en el error masa-concentracion (en sólidos) (item $n^{\circ} \mathrm{s}$ ) parecen disiparse ( 70.5 y $72.7 \%$ respectivamente). Ello nos permite apuntar que, a pesar de la especialización de los estudiantes universitarios, existe una fuerte persistencia de estos errores que habrá que justificar.

Segün nuestra opinión, existe una causa que explica esta gran persistencia del error masa-concentración, causa ya mencionada en la epistemologia genética (Piaget, 1975) y comprobada en el campo de la Fisica (Gil, 1981). Esta causa es el caracter finalista o teleológico del esquema conceptual del alumno en el comportamiento de la materia. Nuestra hipótesis que habrá que constatar más rigurosamente, consiste en suponer que para el alumno el objetivo final de una reaccion quimica es la cantidad de producto obtenido (masa) sin importar como sucede este cambio quimico, de ahi la hegemonia de la cantidad de reaccionantes como la va. riable que realmente importa en todo proceso quimi. co. Recordemos que el uso de la concentración de sus. tancias aparece intimamente relacionado con el modelo elemental de reaccion, organizador previo para poder liegar facimente a la concepción del equilibrio quimico a niveles introductorios, con todas las precaucio. nes que se quieran.

Este error-masa-concentración en las reacciones quimicas, que muchos profesores han observado experimentalmente en sus alumnos, aparece históricamente en el siglo XVIIl; cientificos tan famosos como Berrhollet en su obra "Essai de statique chimique" (Paris,
1803), para poder explicar los dos sentidos posibles de evolución de un sistema quimico, tiene que formular en su tesis sobre las leyes de la afinidad química que "la fuerza absoluta que provocan las reacciones quimicas no sólo depende de las afinidades de cada sus. tancia para combinarse sino que, también, las cantidades de tos cuerpos reaccionantes pueden influir en el sentido de la reacción" (Leicester, 1967). Fué después de casi un siglo cuando es introducido el concepto de masa activa (similar a nuestro concepto actual de concentración) por los noruegos Guldberg y Waage, como consecuencia de su interpretación cinética de la reacción quimica, para que se pueda deducir la conocida ley de acción de masas. Según esta concepción. la fuerza impulsora del proceso es proporcional a la velocidad con que sucede y, en consecuencia, a las concontraciones de los reactivos y no a las masas de las sustancias combinadas (Lindauer, 1962)

\section{DISCUSION}

La diagnosis de los errores conceptuales, aun siendo una etapa necesaria, no tendria sentido si no se bus. can las causas de los mismos y no se ofrece una alter. nativa didáctica con la que erradicar la interpretación no cientifica del comportamiento de la materia.

La persistencia de errores conceptuales en el alumnado que ha sido mostrada en este trabajo preliminar. nos hace suponer que la causa esencial del fracaso de llucstros alumnos en Quimica radica en ja utilizacion de una metodologia empirista que no tiene en cuenta el conocimiento anterior de los alumnos (Bunge. 1478) (Hempel, 1979). Este conocimiento anterior del dium. nado interviene como variable fundamental con la que debe contar todo profesor para una enseñanza slicas de la Quimica (West y Fensham, 1976).

\section{BIBLIOGRAFIA}

AUSU BEL D.P. 1978 "Psicologia educatica. Un punto de visla cog noscitivon (Trillas, México).

BACHELARD G. 1983 «La formation de l'espril scientifique» (Vrin. Parls\}.

BUNGE M. 1978 «Filosofla de la ciencia natural» (Ariel, Barcelona).

CARRASCOSA 1. y GIL D. 1981 aEstudio sobre la persistencia de los errores conceptuales en el aprendizaje de la Fisicas (comuni. cación prescntada en las Jomadas de F. y Q, Alcalá de Henares).
CARRASCOSA J. y GIL D. 1982 "Los errores concepiuales en 12 enseñanza de la Fisica. I Ua estudio de stu persistentia " Prime ras Jornadas de Investigación en Didáctica de F. y $Q$. Valencal

CARRASCOSA J., GIL y GONZALEZ A. 1982 "Un nuevo uala. miento de los errores conceptuales en la enseñanza de la Fisica" (Primeras Jornadas de Investigación en Didactica de $F$ y $O$. Valencia).

GIL. D. 1981 "Evolución de la idea de materia" (1. C.E UniversI. dad Valencia).

GARRET H. E. 197| "Estadistica en Psicología y Educacisn " (Pa. dos, Buenos Aires) 
HEMPEL C.G. 1979 "Filosofía de la Fisica" (Ariel, Barcelona)

JOHNSTONE A.K., MAC DONALD J.J. Y WEBB G. 1977 "Che mical Equilibrium and its conceptual difficulitiesm. (Educ. in Che mistry, 14, 169-171).

KUHN T.S. 197 "La estructura de las tevoluciones cientificas" (Fon. do de Cultura Económica, Méxicol.

LEICESTER H.M. 1967 "Panorama histórico de la Quimica”(A). hambra. Madrid).

LINDAUER M.W. 1962 "The evolution of the concept of Chemicat Equilibrium from 1775 10 1923 * (lour, of Cherm. Ed., 39 (8). 284-390).

NUSSBAUM J. 19R1 "Towards the diagnosis by Science Teachers of pupil's misconceptions: an exercise with student Teachers"
(Eur. J. of Sci. Educ., V. 17, NO. 1, 75.78)

PIACET 3. 1975 alstroducción a la epistemologia gendtica. El pen. samiento lisicon (Paidos. Buenos Aires).

TETERIN H.A. 1979 "Chemical Equilibria and Enetgetics. A terliary viewpoint" (Comunicación presentada en la $3^{*}$ Conferencia Internacional de Ensenanza de la Química, Dublin).

VIGOTSKY L.S. 1973 «Aprendizaje y desarrollo inteletual en la edad escolar" (en "Psicologia y Pedagogla", Akal).

WEST L.H.T. y FENSHAM P.J. 1976 "Prior knowledge or advance organizers as effective variables in chernical learning" (Journ. of Research in Science Teaching. 13 (4). 297.306).

WHEELER A.E. y KASS H. 1978 «Studeni's Misconceptions in Chemical Equilibrium» (Science Education, 62 (2), (223-232).

\section{APENDICE}

\section{Pregunta $n^{\circ} /$}

$\mathrm{El} \mathrm{CaCO}_{3}(\mathrm{~s})$ se descompone por calcinación en $\mathrm{CaO}(\mathrm{s})$ y $\mathrm{CO}_{2}(\mathrm{~g})$ según la ecuación:

$\mathrm{CaCO}_{3}(\mathrm{~s})=\mathrm{CaO}(\mathrm{s})+\mathrm{CO}_{2}(\mathrm{~g})$

Dos experimentadores colocaron la misma cantidad de $\mathrm{CaCO}_{3}(\mathrm{~s})$, uno en una cápsula de porcelana, el otro en un recipiente tapado, como muestran los esquemas adjuntos, y calentaton hasta $900^{\circ} \mathrm{C}$.

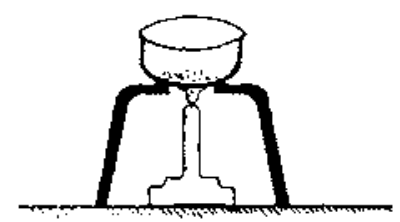

EXРERIMENTO ,

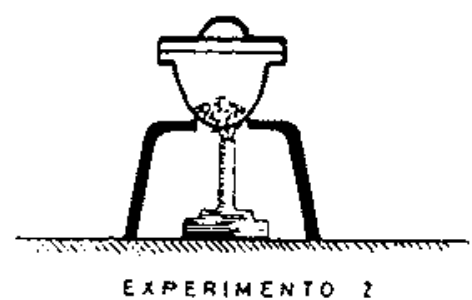

¿Cuál de las siguientes afirmaciones te parece correcta?

a) En ambos experimentos podrá descomponerse todo el $\mathrm{CaCO}_{3}$ inicial.

b) En el experimento 2 podrá descomponerse mayor cantidad de $\mathrm{CaCO}_{3}$ que en el experimento 1.

c) Sólo en el experimento l podrá descomponerse to. do el $\mathrm{CaCO}_{3}$.

d) Sólo en el experimento 2 podrá descomponerse to. do el $\mathrm{CaCO}_{3}$.

e) No lo sé.

\section{Explicación:}

ENSENANZA DE LAS CIENCIAS

\section{Pregunia $n^{\circ} 2$}

El sisterna de la figura ! está en equilibrio a cierta tem. peralura, segun la ecuacion:

$$
\begin{aligned}
& \mathrm{A}(\mathrm{g}) \rightleftarrows \mathrm{B}(\mathrm{g}) \\
& \begin{array}{l}
\text { I MOL OE A } \\
\text { MOL OE a }
\end{array} \\
& \text { FIGURA I. }
\end{aligned}
$$

Dados los siguientes recipientes, a la misma temperatura, en los que A y B están separados como muestran las figuras 2 y 3 :

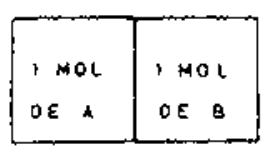

FICURA Z

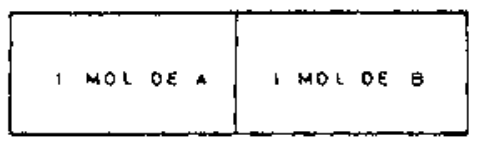

FICURA 3 .

¿Cuál de las siguientes afirmaciones te parece correcta?

a) Los gases A y B están en equilibrio sólo en el recipiente de la figura 2.

b) Los gases A y B están en equilibrio sóto en el recj. piente de la figura 3.

c) En los dos recipientes (2 y 3) los gases A y B están en equilibrio.

d) En ninguno de los dos recipientes ( 2 y 3 ) los gases A y $\mathrm{B}$ están en equilibrio.

e) No lo sé.

Explicación: 
Pregunio no 3

El sistema de la ligura adjunta está en equilibrio se. gún la ecuación $\mathrm{N}_{2} \mathrm{O}_{4}(\mathrm{~g}) \rightleftarrows 2 \mathrm{NO}_{2}(\mathrm{~g})$, para lo cual $\mathrm{K}_{\mathrm{p}}$ viene dada por la expresión:

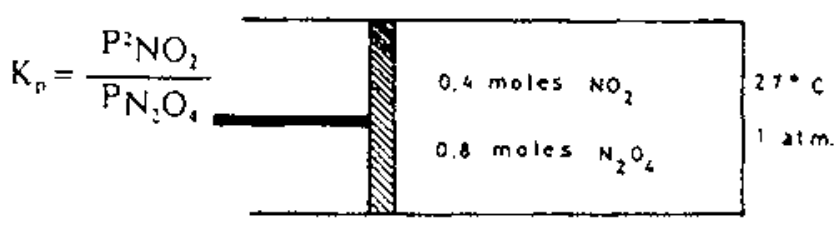

¿Cuál de los siguientes procedimientos es adecuado pa. ra aumentar el valor de $\mathrm{K}_{\mathrm{p}}$ a $27^{\circ} \mathrm{C}$ ?
a) Aumentar la presión tola! (disminuyendo el vo. fumen a temperatura constante).
b) Aumentar la presión parcial del $\mathrm{NO}_{2}$ (añadien- do $\mathrm{NO}_{2}$ al sistema en equilibrio, manteniendo el volumen $y$ la temperatura constantes).
c) Disminuir la presión tolal (aumentando el vo- lumen a lemperatura consiante).
d) La pregunta es absurda.
e) No lo sé.

\section{Expliacion:}

\section{Prtikuman'4}

Una mezcla de $\mathrm{N}_{2}, \mathrm{H}_{2}$ y $\mathrm{NH}_{3}$ está en equilibrio en un recipiente cerrado. según la ecuación:

$$
\mathrm{N}_{2}(\mathrm{~g})+3 \mathrm{H}_{2}(\mathrm{~g}) \rightleftarrows 2 \mathrm{NH}_{3}(\mathrm{~g})
$$

La mezcla en equilibrio contiene: 1.3 moles de $\mathrm{NH}_{3}$

0.1 moles de $\mathrm{N}_{2}$ 0.3 moles de $\mathrm{H}_{2}$
Se desea calcular el valor de la constante de equilibrio, $\mathrm{K}_{\mathrm{c}}$, para dicha reacción. Elige cual de las siguientes respuestas te parece correcta:

a) No es posible el cálcuto.

b) $K_{c}=\frac{0.1 \times 0.31}{1.32}$

c) $K_{c}=\frac{1.32}{0.1 \times 0.3 !}$

d) No lo sé.

Explicación:

Pregunian"s

Al calentar el sólido $\mathrm{NH}_{4} \mathrm{Cl}$, se descompone en los gases $\mathrm{HCl}$ y $\mathrm{NH}_{3}$. Si la reacción se realiza en un recipiente cerrado, se alcanza un estado de equilibrio, según la ecuación:

$$
\mathrm{NH}_{1} \mathrm{Cl}_{(\mathrm{s})} \rightleftarrows \mathrm{H}(\mathrm{I}(\mathrm{g})+\mathrm{NH}, \mathrm{g})
$$

Señala cuál de los siguientes procedimientos te parece adecuado para aumentar la concentración de sotido:
a) Aumentar la presión.
b) Extraer $\mathrm{NH}_{3}$ del recipiente.
c) La pregunta es absurda.
d) Disminuir la presión.
e) No lo sé.

\section{Explicacion:}

Nota.-En el cuestionario pasado a los alumnos de COU y del CAP, habia dos preguntas en cada hoja y no existia ninguna pregunta partida. 